\title{
Electrical nonlinearity in colossal magnetoresistance manganite films: Relevance of correlated polarons
}

\author{
V. Moshnyaga, ${ }^{1}$ K. Gehrke, ${ }^{1}$ O. I. Lebedev, ${ }^{2}$ L. Sudheendra, ${ }^{1}$ A. Belenchuk, ${ }^{3}$ S. Raabe, ${ }^{1}$ O. Shapoval, ${ }^{3}$ J. Verbeeck, ${ }^{2}$ \\ G. Van Tendeloo, ${ }^{2}$ and K. Samwer ${ }^{1}$ \\ ${ }^{1}$ Erstes Physikalicshes Institut, Universität Göttingen, Friedrich-Hund-Platz 1, 37077 Göttingen, Germany \\ ${ }^{2}$ EMAT, University of Antwerp, Groenenborgerlaan 171, B-2020 Antwerpen, Belgium \\ ${ }^{3}$ IEEIT, Academy of Sciences, Str. Academiei 3/3, MD-2028 Chisinau, Republic of Moldova \\ (Received 15 August 2008; revised manuscript received 21 January 2009; published 9 April 2009)
}

\begin{abstract}
The metal-insulator (MI) transition in epitaxial thin films of $\mathrm{La}_{0.75} \mathrm{Ca}_{0.25} \mathrm{MnO}_{3}$ (LCMO) is accompanied by the appearance of an intrinsic electrical nonlinearity. The latter, probed by electrical third harmonic voltage, $U_{3 \omega}$, or resistance, $R_{3 \omega}=d U_{3 \omega} / d J$, is drastically enhanced in the vicinity of the MI transition, $T_{\mathrm{MI}}=267 \mathrm{~K}$. Applied magnetic field, $B=5 \mathrm{~T}$, suppresses the nonlinearity, resulting in a huge "nonlinear" $\mathrm{CMR}_{3 \omega}\left(T_{\mathrm{MI}}\right)$ $\sim 10^{5} \%$. $R_{3 \omega}$ shows a peculiar low-frequency $(\omega \leq 1 \mathrm{kHz})$ dependence, $R_{3 \omega} \sim\left(\omega-\omega_{0}\right)^{n}$, with exponent, $n$, changing across the MI transition from $n \sim 1,5-2$ for $T \geq T_{\mathrm{MI}}$ to $n=1\left(T<T_{\mathrm{MI}}\right)$. The observed electrical nonlinearity in LCMO reflects the behavior of correlated polarons, the number of which dramatically enhances in the vicinity of $T_{\mathrm{MI}}$. We argued that correlated polarons, considered as electric-elastic quadrupoles, provide a nonlinear (quadratic) coupling to the electric field, yielding a third harmonic electric nonlinearity in LCMO. The reference film of $\mathrm{La}_{0.7} \mathrm{Sr}_{0.3} \mathrm{MnO}_{3}$ (LSMO), a prototypic double exchange system with second-order phase transition, is characterized as a linear metallic material in the whole range of temperatures $(T=10-400 \mathrm{~K})$, magnetic fields $(B=0-5 \mathrm{~T})$, and frequencies $(\omega=1-1000 \mathrm{~Hz})$.
\end{abstract}

DOI: $10.1103 /$ PhysRevB.79.134413

PACS number(s): 75.47.Lx, 71.38.-k, 75.47.Gk

\section{INTRODUCTION}

The role of electron-phonon coupling in the localization of electrons, ${ }^{1}$ metal-insulator (MI) transition, and colossal magnetoresistance (CMR) (Ref. 2) in manganites, e.g., $\mathrm{La}_{1-x} \mathrm{Ca}_{x} \mathrm{MnO}_{3}$ (LCMO), is well documented by neutron ${ }^{3-5}$ and X-ray scatterings. ${ }^{6,7}$ In the paramagnetic state $\left(T>T_{C}\right)$ the localization mechanism is provided by Jahn-Teller (JT) lattice distortions, since $\mathrm{Mn}^{3+}$ and $\mathrm{Mn}^{4+}$ have different ionic radius. These static local lattice distortions of $\mathrm{MnO}_{6}$ octahedra with trapped electrons (holes), dubbed as "JT polarons," accommodate within the perovskite crystalline matrix with typically orthorhombic ${ }^{6}$ symmetry in the form of single (lattice) or short-range correlated polarons (CP). The correlation length is estimated to be about $1-3 \mathrm{~nm}^{4}$ and shows no changes with temperature and magnetic field. In addition it was suggested that $\mathrm{CP}$, manifesting themselves as a shortrange ordered $\left(\frac{1}{4}, \frac{1}{4}, 0\right)$ superstructures, originate from the charge and/or orbitally ordered CE phase. ${ }^{4}$ The scattering intensity in neutron and x-ray experiments, assumed to be proportional to the concentration of correlated polarons, $N_{\mathrm{CP}}$, shows a temperature dependence qualitatively similar to that of the resistance, thus reflecting a MI transition. Namely, $N_{\mathrm{CP}}$ is suppressed in the ferromagnetic metallic phase at $T<T_{C}$ and strongly increases by approaching the Curie temperature, $T_{C}$. In the paramagnetic insulating phase, however, $N_{\mathrm{CP}}(T)$ strongly decreases with increasing temperature. ${ }^{6}$ The magnetic field behavior $N_{\mathrm{CP}}(B)$ qualitatively resembles the CMR effect, while even for strong fields $B \sim 5-7 \mathrm{~T}$ there is a measurable $N_{\mathrm{CP}}$ at least for Nd-Sr-Mn-O (NSMO). ${ }^{7}$

The MI transition in manganites is usually studied by means of four-point dc resistive measurements. More rarely ac measurements were performed but no special attention was paid to the electrical nonlinear behavior as a function of temperature and/or magnetic field. However, the ferro/ paramagnetic phase transition in CMR manganites has a peculiar nature as was evidenced by the observation of quasielastic contribution to spin waves, ${ }^{8}$ by optical Brillouin scattering with appearance of a central peak $(\Delta \nu=0)$ at $T$ $\sim T_{C}$ (Ref. 9) and by the change of lattice elasticity ${ }^{10}$ at the phase transition. The peculiar phase transition in manganites, additionally considered within the phase-separation scenario, ${ }^{11}$ needs a more detailed study which accounts for electrical nonlinearity close to the phase transition. A pronounced nonlinear electrical behavior, i.e., nonlinear current-voltage- $J(U)$ characteristics, followed even by a resistance switching, was observed for small-bandwidth manganites such as $\mathrm{Pr}_{0.7} \mathrm{Ca}_{0.3} \mathrm{MnO}_{3}$ (PCMO) (Ref. 12) as well as for NSMO (Ref. 13) in the charge-orbital ordering (COO) regime as well as in the mixed phase region with coexisting $\mathrm{COO}$ and charge-orbital disordered ferromagnetic metallic (FMM) phases. In all these cases the polaron transport is the dominating mechanism and the observed nonlinearity was related to the electric or magnetic field induced "melting" of $\mathrm{COO}$ domains at the expense of the "growing" orbitally disordered FMM phase. ${ }^{14,15}$ The observed huge CMR was then discussed within the phase-separation scenario and percolative phase transition. ${ }^{16}$ For optimally doped LCMO $(x$ $=0.2-0.33)$ with high $T_{C}$, clear MI and CMR behaviors, the nonlinear $J(U)$ characteristics have not been reported to the best of our knowledge. The nonlinearity close to the phase transition was just hypothesized ${ }^{17}$ but no specifications of possible mechanisms, including correlated polarons was suggested.

\section{METHODS}

We studied the nonlinearity by electrical ac third harmonic technique, ${ }^{18}$ which is known to be very sensitive to 
defects in metallic films ${ }^{19}$ as well as to the fluctuations of local temperature and/or charge-carrier concentration as observed for high- $T_{C}$ cuprate thin films close to the superconducting transition. ${ }^{20,21}$ Linear, $U_{\omega}$, and nonlinear third harmonic, $U_{3 \omega}$, ac signals were measured simultaneously using four-probe technique with a "Quantum Design" PPMS system for ac current values $J=1-10^{3} \mu \mathrm{A}$ in the range of frequencies, $\omega=1-10^{3} \mathrm{~Hz}$ and temperatures, $T=10-400 \mathrm{~K}$, and in-plane magnetic fields $B=0-5 \mathrm{~T}$. The low-field $(B=10 \mathrm{mT})$ magnetization was analyzed with a commercial MPMS superconducting quantum interference device (SQUID) system for $T=10-400 \mathrm{~K}$. The structure of the films was characterized by $\mathrm{X}$-ray diffraction (XRD) (Siemens D5000) and transmission electron microscopy (TEM) (Jeol 4000EX and Jeol 3000F). The film samples, prepared by a metalorganic aerosol deposition technique, ${ }^{22,23}$ were individual LCMO (sample C) and LSMO (sample S) layers $(x \sim 0.3)$ as well as $\mathrm{LCMO} / \mathrm{BaTiO}_{3}$ (CB sample) multilayer, all grown on $\mathrm{MgO}(100)$ substrates. Such choice of the film samples was made, on one hand, to compare the behavior of prototypic CMR (LCMO) and double exchange (LSMO) systems and, on another hand, to study the effect of epitaxial misfit strain (CB-film) on the nonlinear behavior.

\section{RESULTS}

The HREM as well as XRD study demonstrate that the films were grown epitaxially on $\mathrm{MgO}(100)$. One can see that the CB multilayer [Fig. 1(a)] as well as C film [Fig. 1(b)] are both of high crystalline quality. The electron-diffraction (ED) pattern obtained from a cross section of the CB-sample [Fig. $1(\mathrm{c})]$ is a superposition of the ED patterns from cubic $\mathrm{MgO}$ substrate and from the tetragonally distorted LCMO and pseudocubic BTO layers. The splitting of the spots due to a different lattice parameters in the layers occurs only along the film growth direction, i.e., normal to the foil set [see inset of Fig. 1(c)]. At the interface the difference in lattice parameter is taken up elastically, yielding a compressive stress in LCMO layer. Moreover, high-resolution TEM (HRTEM) measurements demonstrate coherent and atomically flat interfaces between LCMO and BTO layers [Fig. 1(d)]. A perfect chemical separation of the layer structures has been evidenced by means of energy filtered TEM (EFTEM) technique [Fig. 1(a)]. The color coded image shows the element maps of $\mathrm{Mg}$ (red), Ti (green), and Mn (blue) which clearly coincide with $\mathrm{MgO}, \mathrm{BTO}$, and LCMO regions. According to XRD all the samples $(\mathrm{C}, \mathrm{S}, \mathrm{CB})$ show a pseudocubic perovskite structure, originated from manganite and titanite (CB) phases, but $c$-axis lattice parameters of manganite layers were significantly different. As shown in Table I C and S film possess $c$-axis values very close to those for bulk LCMO and LSMO phases, respectively, indicating a stress-free state in accordance with earlier observations on the films grown on $\mathrm{MgO} .{ }^{23}$ High values of $T_{C}$ and $T_{\mathrm{MI}}$ are the result of stress-free state of $\mathrm{C}$ and $\mathrm{S}$ films. In contrast, the CB-film shows significantly smaller $c$-axis parameter, $c=0.3850 \mathrm{~nm}$, due to a misfit stress, $\varepsilon=\left(C_{\mathrm{LCMO}}-C_{\mathrm{BTO}}\right) /$ $C_{\mathrm{LCMO}} \sim 0.8 \%$, resulting in reduced $T_{\mathrm{MI}}$ and $T_{C}$ (Table I).

In Fig. 2(a) we present the temperature dependences of the third harmonic coefficient, $K_{3 \omega}=\log \left[\left(U_{3 \omega} / U_{\omega}\right)\right]$, which

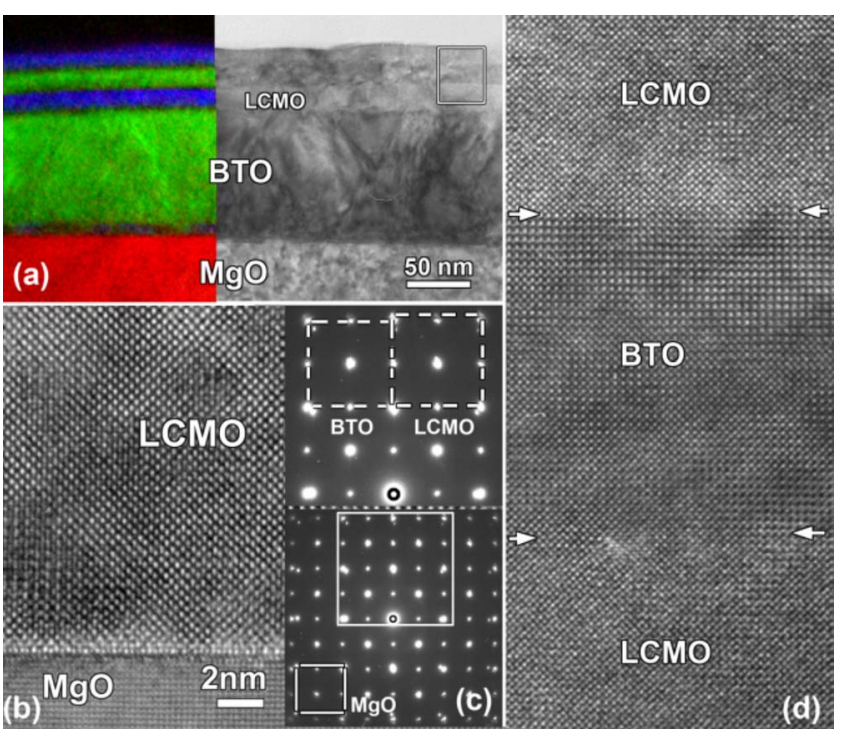

FIG. 1. (Color online) (a) Cross-section low magnification EFTEM (left) and TEM images (right) of CB multilayer film. The following colors are used for chemical mapping: "red" for $\mathrm{Mg}$ ( $\mathrm{MgO}$ substrate), "green"- $\mathrm{Ba}\left(\mathrm{BaTiO}_{3}\right.$ layer $)$ and "blue"- $\mathrm{Mn}$ $\left(\mathrm{La}_{0.7} \mathrm{Ca}_{0.3} \mathrm{MnO}_{3}\right.$ layer); (b) Cross-section HRTEM image of the LCMO film grown on a $\mathrm{MgO}(001)$ substrate; (c) —ED pattern taken from the $\mathrm{CB}$ multilayer film. An enlargement of the region marked by a white rectangle area is given as inset; (d) HRTEM image of a selected area [white rectangle in (a)] area. Note the heteroepitaxial growth of LCMO-BTO layers and the atomically flat and sharp interfaces between the layers (marked by white arrows).

quantifies electric nonlinearity in the $\mathrm{C}$ and $\mathrm{S}$ films. $U_{\omega}$ and $U_{3 \omega}$ are the measured ac voltages on the fundamental, $\omega$, and $3 \omega$ frequencies, respectively. The $S$ film shows the temperature and magnetic field independent $K_{3 \omega}(T, B)$ behavior with a low level of nonlinearity, $K_{3 \omega} \sim-90 \mathrm{~dB}$, comparable to that observed in resistors. In contrast, $\mathrm{C}$ film reveals an increase of nonlinearity up to $K_{3 \omega} \sim-60 \mathrm{~dB}$ with a maximum at $T_{\mathrm{MI}}=267 \mathrm{~K}$. A magnetic field $B=5 \mathrm{~T}$ suppresses nonlinearity close to $T_{\mathrm{MI}}$ down to the level characteristic for the metallic state. Another observation is that for the strained CB film [Fig. 2(b)] $T_{\mathrm{MI}}$ values are reduced both in the $R(T)$ and the $K_{3 \omega}(T)$ curves, reflecting the effect of misfit stress onto linear and nonlinear behaviors. To quantify the nonlinear electric behavior we calculated third harmonic resistance, $R_{3 \omega}=d U_{3 \omega} / d J$, and compared it with the linear resistance, $R_{\omega}=U_{\omega} / J$. In the inset of Fig. 3 one notices very low values of $R_{3 \omega} \sim 10^{-3} \Omega$, observed for the $\mathrm{S}$ film with no additional increase in the nonlinearity at the phase transition, i.e., the $R_{3 \omega}(T)$ apparently scales with $R_{\omega}(T)$ curve. For the LCMO films (C and $\mathrm{CB}$ ) no scaling between $R_{3 \omega}(T)$ and $R(T)$ was observed [Figs. 3(a) and 3(b)]. The third harmonic resistance shows a dramatic increase in the vicinity of $T_{\mathrm{MI}}$, otherwise, both for $T \ll T_{\mathrm{MI}}$ as well as for $T \gg T_{\mathrm{MI}}, R_{3 \omega}$ remains at the very low level, comparable to that of the $S$ film.

The effect of magnetic field on the third harmonic nonlinearity is shown in Fig. 4. To compare the magnetic field behavior in $\mathrm{C}$ and $\mathrm{CB}$ films we present normalized values, $R_{3 \omega}(T) / R_{3 \omega}\left(T_{\mathrm{MI}}\right)$, as a function of the normalized temperature $\mathrm{T} / \mathrm{T}_{\mathrm{MI}}$. One can see that the "nonlinear" MI transition is 
TABLE I. Characteristics of manganite film samples.

\begin{tabular}{lccccc}
\hline \hline Sample & $\begin{array}{c}\text { Thickness } \\
(\mathrm{nm})\end{array}$ & $\begin{array}{c}c \text { axis } \\
(\mathrm{nm}) / \text { stress }(\%)\end{array}$ & $\begin{array}{c}T_{C} \\
(\mathrm{~K})\end{array}$ & $\begin{array}{c}T_{\mathrm{MI}} \\
(\mathrm{K})\end{array}$ & $\begin{array}{c}E_{\mathrm{a}}\left(R_{\omega}\right) \\
(\mathrm{meV})\end{array}$ \\
\hline LSMO(S) & 60 & $0.3890 / 0$ & 358 & 350 & \\
LCMO(C) & 55 & $0.3877 / 0$ & 260 & 267 & 60 \\
LCMO/BTO(CB) & 32 & $0.3850 / 0.8$ & 215 & 210 & 120 \\
\hline \hline
\end{tabular}

extremely sharp in the strain-free $\mathrm{C}$ film and considerably broadened in the strained CB film [Fig. 4(a)]. Remarkably, in the paramagnetic insulating phase a common behavior for different LCMO films was observed. Namely, the nonlinear resistance shows an activated temperature behavior, $R_{3 \omega}(T)$ $\sim \exp \left(-E_{a} / k T\right)$, with similar $E_{a} \approx 0.24-0.28 \mathrm{eV}$. In contrast, the linear resistance, $R_{\omega}(T)$, demonstrates very different and low activation energies, $E_{a}=0.06 \mathrm{eV}$ and $0.12 \mathrm{eV}$, for $\mathrm{C}$ and $\mathrm{CB}$ films, respectively (Table I). In Fig. 4(b) the "nonlinear" $\mathrm{CMR}_{3 \omega}(T)=100 \% \times\left[R_{3 \omega}(T, 0)\right.$ $\left.-R_{3 \omega}(T, 5 \mathrm{~T})\right] / R_{3 \omega}(T, 5 \mathrm{~T})$ is shown. C film exhibits a huge "nonlinear" $\mathrm{CMR}_{3 \omega}=80000 \%$ and a narrow temperature distribution of CMR with full width at half maximum $(\mathrm{FWHM}) \approx 0.05 T_{\mathrm{MI}} \sim 13 \mathrm{~K}$. Moreover the usual or "linear," $\mathrm{CMR}_{\omega}=400 \%$, is apparently much smaller than the nonlinear $\mathrm{CMR}_{3 \omega}$. The strained CB film demonstrates $\mathrm{CMR}_{3 \omega}=700 \%$ with a broad temperature distribution, $\mathrm{FWHM}=0.22 T_{\mathrm{MI}}$ $\sim 50 \mathrm{~K}$. However, the magnitudes of the nonlinear and linear effects are comparable at $T_{\mathrm{MI}}$, i.e., $\mathrm{CMR}_{3 \omega} \approx \mathrm{CMR}_{\omega}$.

In the inset of Fig. 5(a) we present the dependence of the nonlinear voltage, $U_{3 \omega}$, in the $\mathrm{C}$ film on the amplitude of ac current, $J$, at $T=265 \mathrm{~K}$, i.e., very close to $T_{\mathrm{MI}}=267 \mathrm{~K}$. One can see that $U_{3 \omega}(J)$ can be well fitted by a cubic dependence, i.e., $U_{3 \omega} \sim J^{3}$. This illustrates the third harmonic origin of the

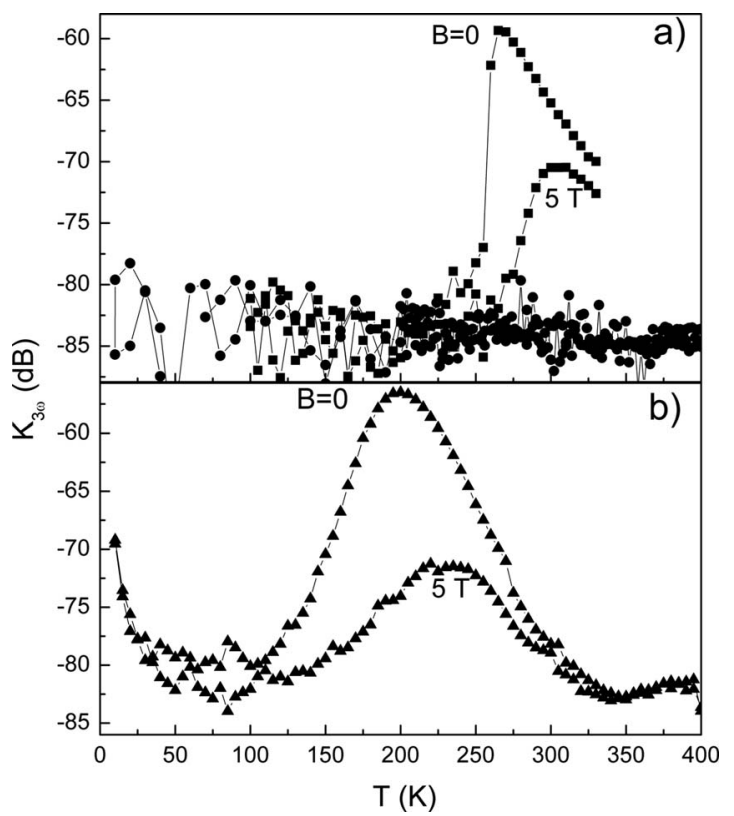

FIG. 2. Temperature dependences of the third harmonic coefficient, $K_{3 \omega}=\log \left[\left(U_{3 \omega} / U_{\omega}\right)\right]$, measured for $J=100 \mu \mathrm{A}, \omega=17 \mathrm{~Hz}$, and magnetic field $B=0$ and $5 \mathrm{~T}$. (a) $\mathrm{C}$ film (squares) and $\mathrm{S}$ film (circles). (b) CB film (triangles). measured nonlinear signal, because with $J=J_{0} \cos \omega t$ one gets $U_{3 \omega} \sim J_{0}^{3} \cos ^{3} \omega t \sim \cos 3 \omega t$ due to trigonometry relations. The nonlinear (current dependent) resistance therefore depends quadratically on the current, $R_{3 \omega}=d U_{3 \omega} / d J \sim J^{2}$. Moreover, $R_{3 \omega}$, shows a peculiar power law frequency dependence [Figs. 5(a) and 5(b)]: $R_{3 \omega}(\omega)=R_{3 \omega}^{*}+A\left(\omega-\omega_{0}\right)^{n}$ in clear contrast to the frequency independent linear resistance $R_{\omega}$ (not shown). Interesting, the exponent, $n$, changes across the phase transition from $n=1$ in the metallic state at $T$ $=200 \mathrm{~K}$ to $n=1.5-2$ in the insulating state $(T \geq 265 \mathrm{~K})$. The development of the insulating phase, monitored by the increase in the nonlinear signal, can be followed by increasing the amplitude of the ac current at a constant temperature, $T=255 \mathrm{~K}$, close but still lower than $T_{\mathrm{MI}}$. As is demonstrated in Fig. 6, a linear frequency dependence of $R_{3 \omega}(\omega)$ is valid for relatively low ac current values, $J \leq 100 \mu \mathrm{A}$. However, for currents $J \geq 200 \mu \mathrm{A}$ the exponent " $n$ " switches to $n$ $=1.5$ and a large $R_{3 \omega}$ values are already seen for very low frequencies. Note that both $n \approx 2$ and large $R_{3 \omega}$ were ob-
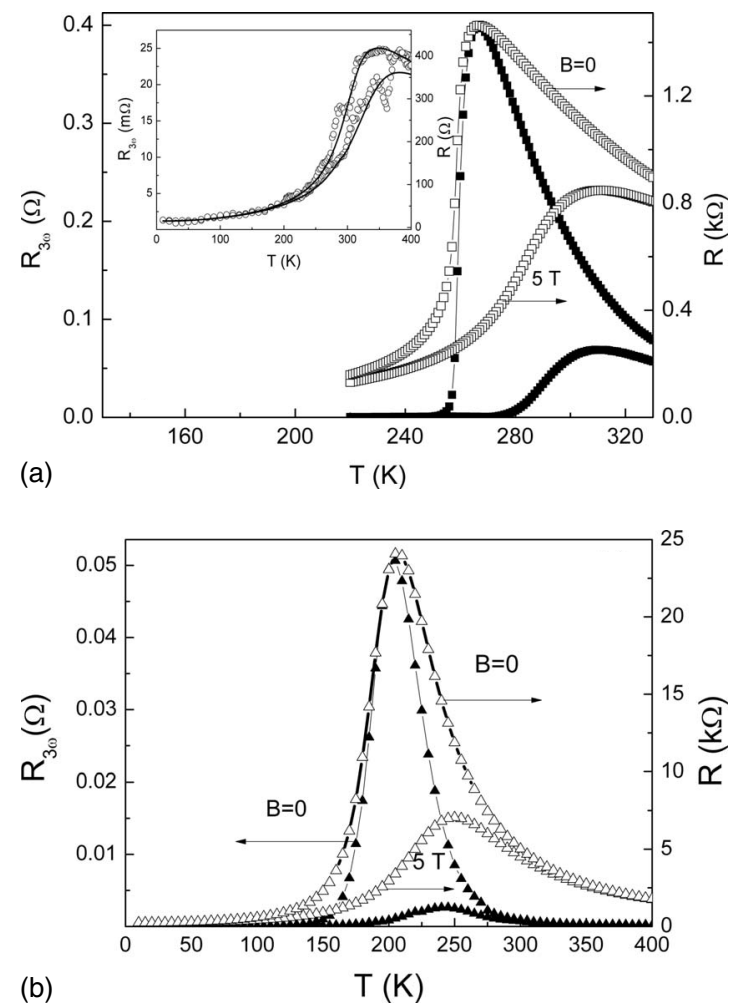

FIG. 3. Temperature dependences of the nonlinear, $R_{3 \omega}(T)$, (left scale, closed symbols), and linear, $R_{\omega}(T)$, (right scale, open symbols) resistances for different samples: (a) C-film $(J=400 \mu \mathrm{A}, \omega$ $=17 \mathrm{~Hz}$ ), and S film [inset in (a)] (b) CB film. 


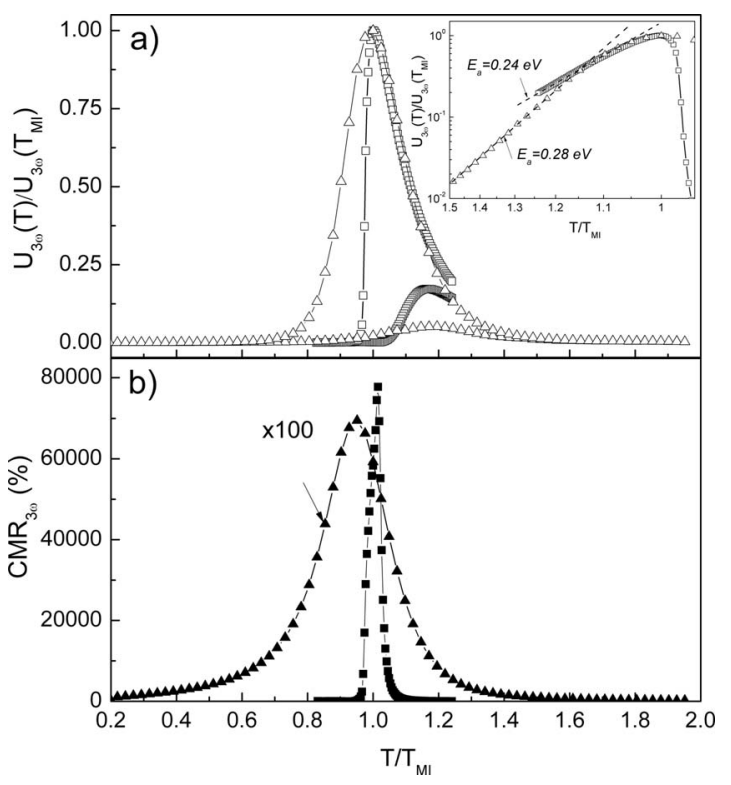

FIG. 4. The dependences of: (a) normalized nonlinear voltage, $U_{3 \omega}(T) / U_{3 \omega}\left(T_{\mathrm{MI}}\right)$, for $B=0$ and $5 \mathrm{~T}$ in $\mathrm{C}$ film (open squares) and $\mathrm{CB}$ film (open triangles) and (b) CMR @ $5 \mathrm{~T}$ in $\mathrm{C}$ film (closed squares) and CB film (closed triangles) as a function of normalized temperature, $T / T_{\mathrm{MI}}$. The inset in (a) illustrates an activated such as $U_{3 \omega}(T)$ behavior for $T>T_{\mathrm{MI}}$ in $\mathrm{C}$ film (squares) and $\mathrm{CB}$ film (triangles).

served for a globally insulating phase for $T>T_{\mathrm{MI}}$ [Figs. 5 and 6]. The difference between metallic and insulating state with respect to the third harmonic electrical nonlinearity is that for a LCMO metal at $T \ll T_{\mathrm{MI}}$ the nonlinear resistance is vanishingly low (comparable with noise) at very low frequencies and currents; moreover, $R_{3 \omega}(\omega)$ grows linearly with increasing frequency. In contrast, for a LCMO insulator $\left(T>T_{\mathrm{MI}}\right)$ the nonlinearity cannot be induced at very low frequencies: the $R_{3 \omega}$ is already relatively large practically at $\omega$ $\sim 0$. In other words there is a gap for exciting nonlinearity in the insulating state and there is no gap (nonlinearity can be induced at almost zero $\omega$ ) in the metallic state.

\section{DISCUSSION}

We would like to point out the remarkably common features with correlated polarons behavior, ${ }^{3-9}$ on the one hand, and the measured electric third harmonic voltage, $U_{3 \omega},\left(R_{3 \omega}\right)$, on the other hand. Namely, they both are: (1) observed in LCMO, but not in LSMO; (2) enhanced close to the phase transition; (3) strongly reduced in the FMM phase for $T$ $\ll T_{C}$ and in the PMI phase ( $T \gg T_{C}$ ); and (4) suppressed in a magnetic field, causing a very large $\mathrm{CMR}_{3 \omega}$ effect. Moreover the observed activation energy, $E_{a} \sim 0.24-0.28 \mathrm{eV}$, of $R_{3 \omega}\left(T>T_{C}\right)$ is very close to the JT deformation energy, $\Delta_{\mathrm{JT}}$ $=0.3 \mathrm{eV},{ }^{24}$ suggesting an universal JT origin of the nonlinearity in different CMR manganite films. Additionally, to the films discussed here in details, we obtained the same values, $E_{a} \sim 0.28,0.29 \mathrm{eV}$, for another LCMO $\left(T_{C}=255 \mathrm{~K}\right)$ and $\left(\mathrm{La}_{5 / 8} \mathrm{Pr}_{3 / 8}\right)_{0.7} \mathrm{Ca}_{0.3} \mathrm{MnO}_{3}\left(T_{C}=194 \mathrm{~K}\right)$ samples, respectively. Based on the fact that static JT distortions do exist in
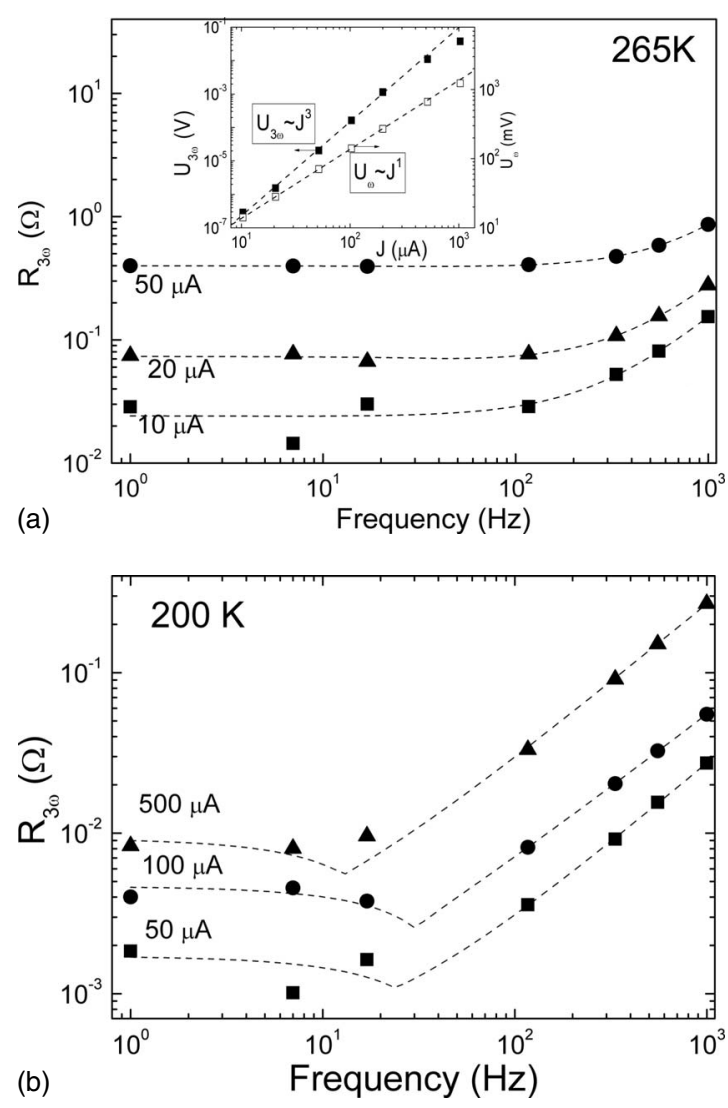

FIG. 5. Frequency dependences of the nonlinear resistance, $R_{3 \omega}$, in $\mathrm{C}$ film, measured at different currents for $T=265 \mathrm{~K}$ (a) and $T=200 \mathrm{~K}$ (b). The symbols represent the data points and dashed lines are the fit curves $R_{3 \omega}=R_{3 \omega}^{*}+A\left(\omega-\omega_{0}\right)^{n}$, with $n=2 \quad(T$ $=265 \mathrm{~K})$ and $n=1(T=200 \mathrm{~K})$. The inset in (a) shows the current dependences of the nonlinear, $U_{3 \omega}$, and linear, $U_{\omega}$, signals at $T$ $=265 \mathrm{~K}$.

LCMO, ${ }^{3-7}$ hosting within an orthorhombic $\left(P_{n \text { ma }}\right)$ structure, and do not in LSMO $(R-3 c)$, it looks reasonable to relate the electric third harmonic nonlinearity to correlated polarons.

The possible mechanism for the contribution of correlated polarons to the electrical third harmonic voltage can be deduced from the observed quadratic dependence of $R_{3 \omega}$ on the electric current (field), i.e., $R_{3 \omega}(J) \sim J^{2}$. In other words one should address the origin of a nonlinear coupling of electric field to the JT distortions, i.e., correlated polarons. Unlikely such coupling occurs through a dipole mechanism; no dipoles and no ferroelectricity was detected in the manganites to the best of our knowledge. However, a quadrupole mechanism looks probable because of several reasons. In general, Mn ions with electrons on $d$ orbitals $(L=2)$ provide a natural example of the quadrupolar charge distribution. In details, a correlated JT polaron, considered as a short-range correlated $(\sim 1 \mathrm{~nm})$ region with CE-type ordering of $\mathrm{Mn}^{3+} / \mathrm{Mn}^{4+}$ ions, ${ }^{4}$ can be viewed as an electric quadrupole with ordered positive $\left(\mathrm{Mn}^{4+}\right)$ and negative $\left(\mathrm{Mn}^{3+}\right)$ charges in the corners of square with a side along the (110) axis of a pseudocubic perovskite. Naturally, an ideal long-range ordered CE-phase seems to be quadrupole free- the $\mathrm{Mn}^{3+} / \mathrm{Mn}^{4}$ charge imbalance is fully compensated and the charge distribution seems to be completely spherically symmetric. But for the short- 


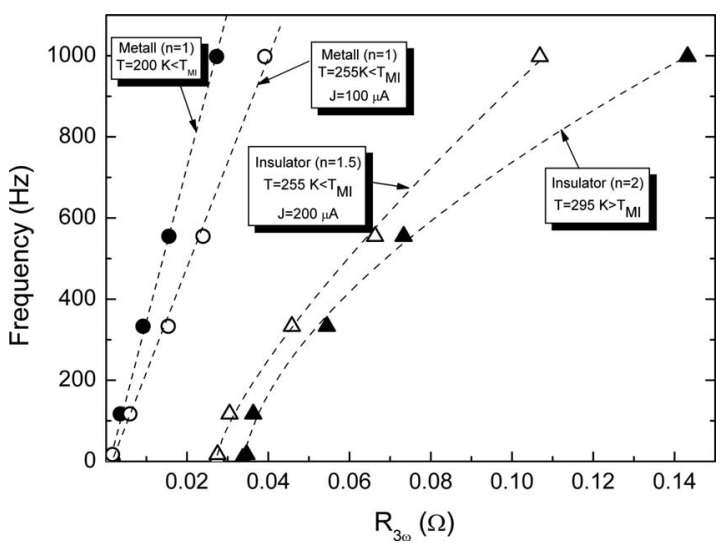

FIG. 6. Current (field) induced metal-insulator transition in C film at $T=255 \mathrm{~K}<T_{\mathrm{MI}}$ as elucidated from the frequency dependence of nonlinear resistance (open circles relate to $J=100 \mu \mathrm{A}$ and open triangles for $J=200 \mu \mathrm{A}$ ). The exponent " $n$ " in the frequency dependence of $R_{3 \omega}$ changes from a $n=1$ to $n=1.5$ with increasing the amplitude of ac current. For comparison the data for the metallic state at $T=200 \mathrm{~K}$ with $n=1$ (closed circles) and for the insulating state $T=295 \mathrm{~K}$ with $n=2$ (closed triangles) are also presented. To present all the data on the same graph the $R_{3 \omega}$ values at $T=255 \mathrm{~K}$ for $J=100 \mu \mathrm{A}$ (open circles) and for $J=200 \mu \mathrm{A}$ (open triangles) were divided by factor 7 and 15 , respectively.

range ordered CE-regions a charge asymmetry of a quadrupolar origin due to ellipticity is possible. Even if the charges are not exclusively located at Mn sites but rather are shifted along Mn-O-binding length ${ }^{15}$ (Zener polatrons) a similar quadrupolar presentation may be valid, assuming the CEphase geometry.

It is known from classical nonlinear optics ${ }^{25}$ that a quadrupole moment, $Q$, does couple to an external a.c. electric field, yielding a form, $Q=\chi E^{2}$, with $\chi$ quarupolar susceptibility. This means that a quadrupolar moment can be also induced by an external electric field. Formally, the development of the resistance as a row on exponents of electric field should start from the term $\sim E^{2}$ (the linear and other odd terms are unphysical because they would give a negative resistance), yielding: $R(E) \approx R_{\omega}+A E^{2}+\cdots(\cdots)^{*} E^{2 n}$. Here the second term corresponds to the third harmonic nonlinear resistance (see the last paragraph of Sec. III). Viewed as a nonlinear interaction of an external a.c. electric field with quadrupoles, the $R_{3 \omega}$ is then proportional to the number of quadrupoles in the volume of the sample, i.e., to the concentration of correlated polarons, $N_{\mathrm{CP}}$, and can be written as: $R_{3 \omega}=A N_{\mathrm{CP}}(Q / \chi)$. Thus, the third harmonic nonlinearity in LCMO can be rationalized within the nonlinear coupling of electric field to the correlated polarons, assuming them as electric quadrupoles. An enhancement of the nonlinearity at the transition region $\left(T \sim T_{C}\right)$ can be naturally explained within the temperature dependent concentration of quadrupoles (polarons), $N_{\mathrm{CP}}(T)$. In the ferromagnetic metallic state, $T<T_{C}$, the nonlinear signal, $U_{3 \omega}$, is strongly suppressed to the very low level $\sim-80-90 \mathrm{~dB}$, which exceeds nevertheless the noise level, $\sim-100 \mathrm{~dB}$. However, the nonlinearity does not disappear completely, indicating that $N_{\mathrm{CP}}\left(T<T_{C}\right)$ is small but final. The nonlinearity can be recovered, meaning that correlated polarons as quadrupoles can be excited by increasing current (field) even in the metallic state (see Fig. 5). Remarkably, a third harmonic MI transition can be also switched in the globally metallic state at $255 \mathrm{~K}<T_{\mathrm{MI}}$ (Fig. 6). These results strongly support recent experimental ${ }^{26}$ and theoretical ${ }^{17}$ studies, which stated that correlated polarons do survive in the metallic state.

Very large difference between the values of $R_{\omega}$ and $R_{3 \omega}$ as well as different temperature dependences $R_{\omega}(T)$ and $R_{3 \omega}(T)$ dependences indicate, probably, that different electronic systems may contribute to these quantities. We believe that nonlinear resistance, $R_{3 \omega}$, related to correlated polarons according to the proposed quadrupolar mechanism seems, to be more sensitive and thus more informative for the study of MI transition. Namely, the transition looks extremely sharp (Fig. 3 ), the $\mathrm{CMR}_{3 \omega} \sim 10^{5} \%$ (Fig. 4) is indeed a "colossal" effect and, finally, the activation energy in $R_{3 \omega}(T)$ dependence is very close to the JT deformation energy. This is consistent with the proposed origin of nonlinearity, $R_{3 \omega} \sim N_{\mathrm{CP}}$, which is caused exclusively by the electrons trapped by short-range correlated JT distortions. However, the "puzzle" is that only a small part of electrons are trapped by JT distortions, i.e., $N_{\mathrm{CP}} \ll N$, where $N$ is an overall number of carrier due to $\mathrm{Ca}$ doping. The carriers, mostly behaving as itinerant or band electrons (even if the bandwidth is small), are influenced by the random potential fluctuations due to defects and disorder. $^{27,28}$ That is, we believe, the reason why $R_{\omega}$, reflecting these itinerant electrons, shows a smeared transition, smaller CMR and smaller activation energy, $E_{a}$ $\sim 0.06-0.1 \mathrm{eV}$. We do not contradict the $R_{\omega}$ and $R_{3 \omega}$ approaches but we would like to point out that $R_{3 \omega}$ seems to be more adequate and more sensitive to study the intrinsic lattice effects (correlated polarons), which are enhanced in the vicinity of the phase transition and, thus, extremely sensitive to the temperature as well as to electric and magnetic fields.

One can make an estimation of the relative concentration of correlated polarons, $N_{\mathrm{CP}} / N$, basing on the measured temperature dependence $K_{3 \omega}(T)=R_{3 \omega}(T) / R_{\omega}(T)$ and considering the following simple phenomenological description. Very generally, the overall resistance of a manganite can be expressed as

$$
R_{\Sigma} \sim \frac{1}{N-N_{\mathrm{CP}}}
$$

Here, $N$ and $N_{\mathrm{CP}}$ are the concentrations of the charge carriers, and correlated polarons, i.e., trapped or localized electrons. The latter do not contribute more to the conductivity and lead to the increase in the overall resistance. The next assumption is that the concentration of correlated polarons is small, i.e., $N_{\mathrm{CP}} \ll N$. This looks reasonable because both neutron and $\mathrm{x}$-ray scattering data emphasize a small number of correlated polarons in LCMO.$^{3-7}$ Then one gets from Eq. (1),

$$
R_{\Sigma} \sim \frac{1}{N\left(1-\frac{N_{\mathrm{CP}}}{N}\right)} \approx \frac{1}{N}\left(1+\frac{N_{\mathrm{CP}}}{N}\right) \sim \frac{1}{N}+\frac{N_{\mathrm{CP}}}{N^{2}} .
$$

Taking into account the ac measurements, the first and dominating term will correspond to $R_{\omega}$ and the second and much smaller term $\left(N_{\mathrm{CP}} \ll N\right)$ is then proportional to $R_{3 \omega}$ due to the 


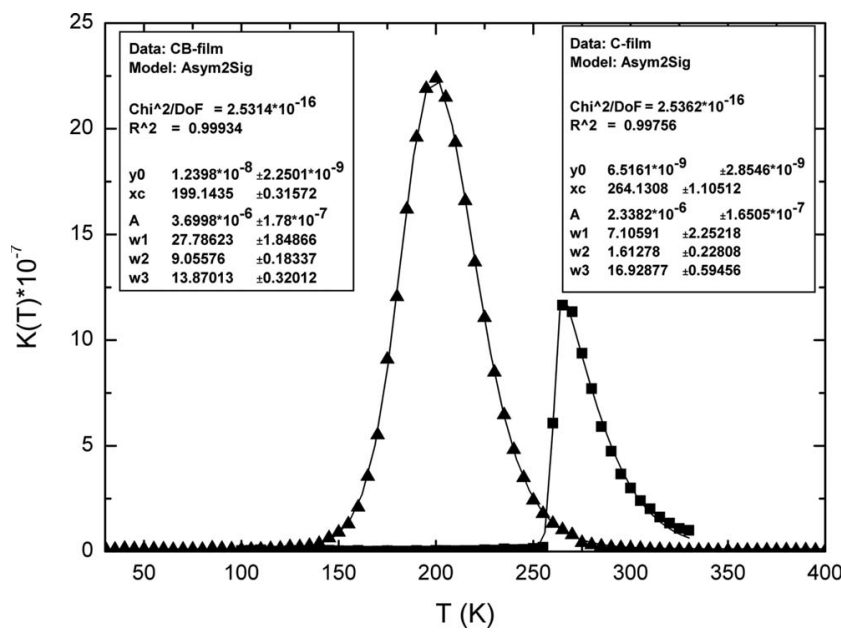

FIG. 7. The measured $K_{3 \omega}(T)=R_{3 \omega}(T) / R_{\omega}(T)$ dependence in C film (squares) and in CB film (triangles) and the fitting made by means of asymmetric double sigmoid function [see Eqs. (6) and (7)], shown as lines.

discussed above nonlinear coupling of correlated polarons to electric field, i.e., $R_{3 \omega} \sim N_{\mathrm{CP}}$. Then $R_{\Sigma}$ and the ratio, $K$ $=R_{3 \omega} / R_{\omega}$, we are looking for, is given by

$$
K \equiv \frac{R_{3 \omega}}{R_{\omega}}=\frac{N_{\mathrm{CP}} \times N}{N^{2}}=\frac{N_{\mathrm{CP}}}{N} .
$$

The temperature dependent concentration of correlated polarons, $N_{\mathrm{CP}}(T)$, should be proportional to the overall concentration of carriers, $N(T)$, and can be described by the following formula:

$$
N_{\mathrm{CP}}(T)=N(T) \alpha(T)=N(T) \alpha_{0}[1+\beta(T)] .
$$

The function $\alpha(T)<1$ describes the probability of an electron to be trapped by a JT distortion, which in principle is determined by the strength of the electron-phonon coupling and depends on the temperature and, probably, on other control parameters, such as doping, magnetic field, and pressure. To illustrate the effect of the phase transition the trapping probability in Eq. (4) was separated into a temperature independent, $\alpha_{0}$, and temperature dependent, $\beta(T)$, parts. Then the ratio $\mathrm{K}$ can be rewritten as

$$
K=\alpha_{0}[1+\beta(T)]
$$

The experimentally measured ratio, $K_{\exp }(T)=R_{3 \omega} / R_{\omega}$, for $\mathrm{C}$ and CB films (see Fig. 7) can be fitted remarkably good $\left(R^{2}=0.998\right)$ by the following analytic expression, which shows the same functional form as Eq. (5). Namely,

$$
K_{\text {exp }}(T) \equiv \frac{R_{3 \omega}}{R_{\omega}}=K_{0}[1+A f(T)] .
$$

Here the constant, $K_{0} \sim 10^{-8}-10^{-9}$, represents the ratio $R_{3 \omega} / R_{\omega}$ at $T<T_{\mathrm{MI}}$, describing a very small degree of the nonlinearity and very small amount of correlated polarons in the metallic phase: $K\left(T<T_{\mathrm{MI}}\right)=\alpha_{0}$. The fitting function, $f(T)$, is the so-called "asymmetric double sigmoid" (ADS) function:

$$
\begin{aligned}
f(T)= & \left\{\frac{1}{1+\exp \left[\frac{\left.-\left(T-T_{\mathrm{MI}}+\frac{w_{1}}{2}\right)\right]}{w_{2}}\right]}\right\} \\
& \times\left\{1-\frac{1}{1+\exp \left[\frac{-\left(T-T_{\mathrm{MI}}-\frac{w_{1}}{2}\right)}{w_{3}}\right]}\right\}
\end{aligned}
$$

The values of ADS belong to the interval $[0,1]$ for $T \in\left[T_{\mathrm{MI}}\right.$ $\left.-\mathrm{w}_{1} ; T_{\mathrm{MI}}+w_{1}\right]$. All the fit parameters are given in Table II. Here we note that, $w_{2} \neq w_{3}$, are the widths of the two transition regions at the left and right sides from $T_{\mathrm{MI}}$, respectively. Another constant in the fitting function, $A \sim 300$, describes the maximal value of $K_{\exp }$ at $T=T_{\mathrm{MI}}$, giving us finally an estimation of the maximal fraction $\left(N_{\mathrm{CP}} / N\right)$ of correlated polarons close to $T_{\mathrm{MI}}$,

$$
\alpha\left(T_{\mathrm{MI}}\right)=K_{0} \times A \approx(1-2) \times 10^{-6} .
$$

One can see that this number is very small and qualitatively is in accordance to neutron and x-ray scattering data ${ }^{3-7}$ on single crystals of LCMO with Ca-doping, $x=0.2$ and 0.3 . Taking into account that our $\mathrm{C}$ film $(x \sim 0.25)$ is grown in a strain-free state on $\mathrm{MgO}(100)$, a comparison with single crystals looks reasonable. Note that the concentration of polarons seems to be not strongly affected by the epitaxy strain as we can see that even for a strained CB film this number is also relatively small.

We would like to outline another important aspect of the above model. The fact that the best fit of the measured nonlinearity ratio [Eq. (6)] in LCMO is given by the ADS function (7) indicates that, indeed, two independent processes control the temperature evolution of correlated polarons as well as of the CMR effect, both mainly limited to the vicinity of the phase transition. Namely, the first process, quantified by the transition width $w_{3}$, contributes to the increase of $N_{\mathrm{CP}}$ by $T>T_{\mathrm{MI}}$ and thus should be related to the increase of $\mathrm{COO}$ tendency as the temperature decreases. The second $w_{2}$ process, manifested by a sharp drop of $N_{\mathrm{CP}}$ at the left side of MI

TABLE II. Fitting parameters in the $K_{3 \omega}(T)$ function.

\begin{tabular}{lcccccc}
\hline \hline Sample & $K_{0}$ & $\mathrm{~A}$ & $\begin{array}{c}T_{\mathrm{MI}} \\
(\mathrm{K})\end{array}$ & $w_{1}$ & $w_{2}$ & $w_{3}$ \\
\hline LCMO(C) & $7 * 10^{-9}$ & 360 & 264 & 7.1 & 1.6 & 16 \\
LCMO/BTO(CB) & $1.1 * 10^{-8}$ & 339 & 199 & 27.8 & 9 & 14 \\
\hline \hline
\end{tabular}


transition, is definitely caused by the long-range ferromagnetic ordering due to double exchange interaction. Note, the $w_{3}$ values for $\mathrm{C}$ and $\mathrm{CB}$ films are comparable to each other (see Table II), but the $w_{2}$ values are very different (1.6 and 9). This indicates that the temperature evolution of correlated polarons (COO) seems to be an universal intrinsic behavior, i.e., it is less dependent on the sample quality. In contrast, ferromagnetic transition is, indeed, strongly influenced by extrinsic effects of strain and disorder.

Very interesting is the observed frequency dependence of the nonlinear resistance in LCMO, i.e., $R_{3 \omega} \sim\left(\omega-\omega_{0}\right)^{n}$ [Figs. $5(\mathrm{a})$ and 5(b)]. Within the proposed quadrupolar model it can be interpreted by the frequency dependent quadrupolar susceptibility, i.e., $\chi(\omega) \sim\left(\omega-\omega_{0}\right)^{-n}$. Considering very lowfrequency range $(\omega=1-1000 \mathrm{~Hz})$ of ac current, such behavior seems to be not directly related to any quasiparticle excitations (phonons, electrons, or magnons) but rather represents the response of the crystalline lattice as a whole to ac electric field. In that sense it looks reasonably to suppose that $\mathrm{CP}$, excited by ac electric field, modify elastic properties of the crystalline lattice, yielding to its softening, particularly at the phase transition. This leads to a nonlinear electrical response, which depends on the rate of electric stimuli as in a glassy material. The coupling of electrons to the lattice strain, which is necessary to induce such a softening, can be also rationalized within the quadrupole mechanism, considering that correlated polarons, composed of $\mathrm{Mn}^{3+} / \mathrm{Mn}^{4+}$ species, can be viewed both as electric and also elastic quadrupoles - the difference of ionic radii of these manganese ions induces a local lattice strain distribution of the quadrupolar origin. ${ }^{23}$ Recently, the importance of both electric $^{29}$ and elastic ${ }^{10}$ quadrupolar effects for the formation of stripe phases as a form of charge-orbital ordering in LCMO was elucidated. Moreover, it was also argued that the quadrupolar lattice contribution dominates magnetization induced optical second-harmonic nonlinearity in LCMO. ${ }^{30}$

A slow resistance dynamics was reported earlier in PCMO (Refs. 31 and 32) and interpreted within the reorganization of
CP or charge-density waves. Here we note that LCMO close to phase transition also possesses a nonlinear polaronic glass properties. The transition itself is manifested by the change in the exponent from $n=1$ for $T<T_{\mathrm{MI}}$ in the metallic state to $n=1.5 / 2$ for $T>T_{\mathrm{MI}}$ (insulator). It is interesting that percolative MI transition in artificial metal-insulator composites $^{33,34}$ is also accompanied by a change in exponent in power-law frequency dependence of the ac conductance. However, in contrast to the nonlinear resistance in LCMO, the composites show: (a) a decrease in the ac resistance with decreasing frequency (this is also valid for PCMO) and (b) that $n \sim 2$ is observed for a percolative (metallic) and $n=1$ for a nonpercolative (insulating) regime. Such behavior, well described within an equivalent RC circuit, apparently cannot be adopted for the nonlinearity in LCMO. This also supports a "lattice origin" of the observed nonlinearity in LCMO, but the detailed mechanism is not clear up to now.

In conclusion, we demonstrated that large CMR is intimately coupled with electric nonlinearity as observed in LCMO. The minor CMR in case of LSMO is in line with its linear electric behavior. All this is naturally related to the existence of correlated polarons close to the first-order phase transition in LCMO and their absence in LSMO (secondorder transition). However, the concentration of correlated polarons, $N_{\mathrm{CP}} / N$, seems to be surprisingly small, thus still "puzzling" their microscopic role in the CMR effect. We believe our data bring new insights on the correlated polarons and the role of low-frequency dynamics in CMR and percolative phase transition in manganites.

\section{ACKNOWLEDGMENTS}

The authors thank E. Dagotto for useful comments and W. Felsch for reading of the paper and discussions. Deutsche Forschungsgemeinschaft via Project No. SFB 602, TPA2 is acknowledged. O.I.L, J.V., and G.V.T. acknowledge the financial support of the European Union under the Framework 6 program under a contract for an Integrated Infrastructure Initiative, Reference 026019 ESTEEM.
${ }^{1}$ A. J. Millis P. B. Littlewood, and B. I. Shraiman, Phys. Rev. Lett. 74, 5144 (1995).

${ }^{2}$ R. von Helmolt, J. Wecker, B. Holzapfel, L. Schultz, and K. Samwer, Phys. Rev. Lett. 71, 2331 (1993).

${ }^{3}$ S. Shimomura, N. Wakabayashi, H. Kuwahara, and Y. Tokura, Phys. Rev. Lett. 83, 4389 (1999).

${ }^{4}$ C. P. Adams, J. W. Lynn, Y. M. Mukovskii, A. A. Arsenov, and D. A. Shulyatev, Phys. Rev. Lett. 85, 3954 (2000).

${ }^{5}$ P. Dai, J. A. Fernandez-Baca, N. Wakabayashi, E. W. Plummer, Y. Tomioka, and Y. Tokura, Phys. Rev. Lett. 85, 2553 (2000).

${ }^{6}$ V. Kiryukhin, T. Y. Koo, H. Ishibashi, J. P. Hill, and S.-W. Cheong, Phys. Rev. B 67, 064421 (2003).

${ }^{7}$ Z. Koo, V. Kiryukhin, P. A. Sharma, J. P. Hill, and S.-W. Cheong, Phys. Rev. B 64, 220405 (2001).

${ }^{8}$ J. W. Lynn, R. W. Erwin, J. A. Borchers, Q. Huang, and A. Santoro, Phys. Rev. Lett. 76, 4046 (1996).

${ }^{9}$ Md. M. Seikh, C. Narayana, L. Sudheendra, A. K. Sood and C.
N. R. Rao, J. Phys.: Condens. Matter 16, 4381 (2004).

${ }^{10}$ D. I. Khomskii and K. I. Kugel, Phys. Rev. B 67, 134401 (2003).

${ }^{11}$ E. Dagotto et al., Phys. Rep. 344, 1 (2001).

${ }^{12}$ S. Mercone, A. Wahl, Ch. Simon, and C. Martin, Phys. Rev. B 65, 214428 (2002).

${ }^{13}$ N. Biškup, A. de Andrés, I. M. Ochando, and M. T. Casais, Phys. Rev. B 73, 184404 (2006).

${ }^{14}$ A. Asamitsu, Y. Tomioka, H. Kuwahara, and Y. Tokura, Nature (London) 388, 50 (1997).

${ }^{15}$ Ch. Jooss, L. Wu, T. Beetz, R. F. Klie, M. Beleggia, and M. A. Schofield, Proc. Natl. Acad. Sci. U.S.A. 104, 13597 (2007).

${ }^{16}$ J. Burgy, M. Mayr, V. Martin-Mayor, A. Moreo, and E. Dagotto, Phys. Rev. Lett. 87, 277202 (2001).

${ }^{17}$ Sen, G. Alvarez, and E. Dagotto, Phys. Rev. Lett. 98, 127202 (2007).

${ }^{18}$ V. Peterson and P.-O. Harris, Electronics 39, 93 (1966). 
${ }^{19}$ Q. Wen and D. R. Clarke, J. Appl. Phys. 83, 1132 (1998).

${ }^{20}$ J. C. Booth, J. A. Beall, D. A. Rudman, L. R. Vale, and R. H. Ono, J. Appl. Phys. 86, 1020 (1999).

${ }^{21}$ J. G. Ossandon, S. Sergeenkov, P. Esquinazi, and H. Kempa, Supercond. Sci. Technol. 18, 325 (2005).

${ }^{22}$ V. Moshnyaga, I. Khoroshun, A. Sidorenko, P. Petrenko, A. Weidinger, M. Zeitler, B. Rauschenbach, R. Tidecks, and K. Samwer, Appl. Phys. Lett. 74, 2842 (1999).

${ }^{23}$ V. Moshnyaga, L. Sudheendra, O. I. Lebedev, S. A. Köster, K. Gehrke, O. Shapoval, A. Belenchuk, B. Damaschke, G. van Tendeloo, and K. Samwer, Phys. Rev. Lett. 97, 107205 (2006).

${ }^{24}$ D. S. Dessau and Z.-X. Shen, in Colossal Magneto-resistive Oxides, edited by Y. Tokura (Gordon and Breach Science, New York, 2000).

${ }^{25}$ D. S. Bethune, R. W. Smith, and Y. R. Shen, Phys. Rev. A 17, 277 (1978).

${ }^{26}$ N. Mannella, W. L. Yang, X. J. Zhou, H. Zheng, J. F. Mitchell, J.
Zaanen, T. P. Devereaux, N. Nagaosa, Z. Hussain, and Z.-X. Shen, Nature (London) 438, 474 (2005).

${ }^{27}$ V. Moshnyaga, S. Klimm, R. Tidecks, S. Horn, and K. Samwer, J. Magn. Magn. Mater. 211, 167 (2000).

${ }^{28}$ P. Orgiani, C. Adamo, C. Barone, A. Galdi, A. Yu. Petrov, D. G. Schlom, and L. Maritato, Phys. Rev. B 76, 012404 (2007).

${ }^{29}$ L. Sudheendra, V. Moshnyaga, E. D. Mishina, B. Damaschke, Th. Rasing, and K. Samwer, Phys. Rev. B 75, 172407 (2007).

${ }^{30}$ E. D. Mishina, A. I. Morosov, A. V. Mishina, V. Moshnyaga, L. Sudheendra, K. Samwer, and Th. Rasing, Phys. Rev. B 75, 064401 (2007).

${ }^{31}$ A. Anane, J.-P. Renard, L. Reversat, C. Dupas, P. Veillet, and M. Viret, Phys. Rev. B 59, 77 (1999).

${ }^{32}$ N. Kida and M. Tonouchi, Phys. Rev. B 66, 024401 (2002).

${ }^{33}$ Y. Song, T. W. Noh, S.-I. Lee, and J. R. Gaines, Phys. Rev. B 33, 904 (1986).

${ }^{34}$ K. Sen and A. K. Gupta, Phys. Rev. B 59, 9167 (1999). 